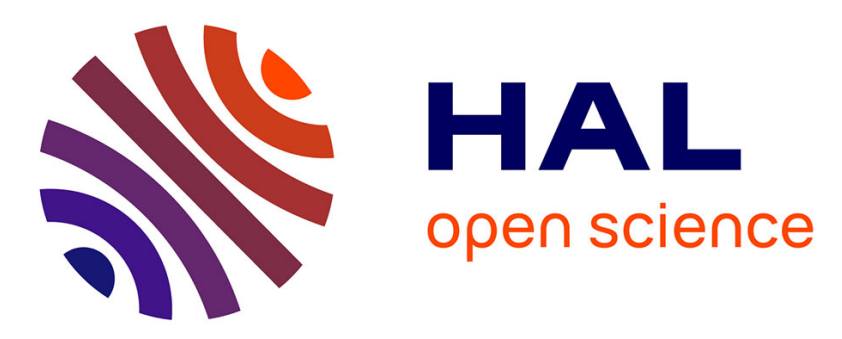

\title{
Run-time Coarse-Grained Hardware Mitigation for Multiple Faults on VLIW Processors
}

Rafail Psiakis, Angeliki Kritikakou, Olivier Sentieys, Emmanuel Casseau

\section{To cite this version:}

Rafail Psiakis, Angeliki Kritikakou, Olivier Sentieys, Emmanuel Casseau. Run-time Coarse-Grained Hardware Mitigation for Multiple Faults on VLIW Processors. DASIP 2019 - Conference on Design and Architectures for Signal and Image Processing, Oct 2019, Montréal, Canada. pp.1-6. hal-02344282

\section{HAL Id: hal-02344282 https://hal.inria.fr/hal-02344282}

Submitted on 4 Nov 2019

HAL is a multi-disciplinary open access archive for the deposit and dissemination of scientific research documents, whether they are published or not. The documents may come from teaching and research institutions in France or abroad, or from public or private research centers.
L'archive ouverte pluridisciplinaire HAL, est destinée au dépôt et à la diffusion de documents scientifiques de niveau recherche, publiés ou non, émanant des établissements d'enseignement et de recherche français ou étrangers, des laboratoires publics ou privés. 


\title{
Run-time Coarse-Grained Hardware Mitigation for Multiple Faults on VLIW Processors
}

\author{
Rafail Psiakis, Angeliki Kritikakou, Olivier Sentieys, Emmanuel Casseau \\ Univ Rennes, INRIA, CNRS, IRISA, France \\ Email: \{emmanuel.casseau, angeliki.kritikakou\}@irisa.fr
}

\begin{abstract}
As transistors scale down, processors are more vulnerable to radiation that can cause multiple transient faults in function units. Rather than excluding these units from execution, performance overhead of VLIW processors can be reduced when fault-free components of these affected units are still used. In the proposed approach, the function units are enhanced with coarse-grained fault detectors. A re-scheduling of the instructions is performed at run-time to use not only the healthy function units, but also the fault-free components of the faulty function units. The scheduling window of the proposed mechanism is two instruction bundles being able to explore mitigation solutions in the current and the next instruction execution. Experiments show that the proposed approach can mitigate a large number of faults with low performance and area overheads.
\end{abstract}

\section{INTRODUCTION}

The energy transferred by radiation is known to cause transient faults on system hardware, which may severely affect the application execution. As technology size decreases, the hardware becomes more and more susceptible to radiation. Although multiple simultaneous faults have been neglected for a long time, they can no longer be negligible for technologies of 130nm and beyond [1]. A single particle hitting the silicon creates secondary particles, which can be emitted in several directions, and, thus, affect different nodes of a circuit [2].

Approaches to deal with multiple faults either apply hardware redundancy or software redundancy. Hardware redundancy inserts spare resources to the original processor to execute in parallel the same instruction and to compare the obtained results [3]. Small performance overhead is usually observed (due to the need of comparison), whereas the area overhead is significant. Software redundancy modifies the program by inserting redundant instructions to be executed on the original processor. Although the area is not increased, the impact on the execution time is significant due to the execution of redundant and comparison instructions [4]. However, when the system processor has parallel Function Units (FUs), the idle FUs can be used to execute these instructions. Such type of processors are the Very Long Instruction Word (VLIW) processors, which usually consist of complex FUs able to execute all types of operations and simpler ones that cannot execute sophisticated operations, such as multiplications and divisions. Existing approaches on VLIW processors that detect the faulty units before the application execution, such as schedule multi-versioning [5] and instruction modification [6], cannot be applied for soft errors. Few approaches detect the faulty FUs during execution. However, instruction duplication and re-execution approaches [7] and run-time rescheduling mechanisms [8] have not been explored for multiple errors. The work in [9] applies duplication and triplication of the instructions for multiple errors, whereas a mechanism is proposed to exclude the faulty FUs permanently for the rest of the execution. However, the negative impact on performance is significant.

To reduce this performance degradation while keeping the area overhead low, we propose a coarse-grained hardware mechanism that detects the faults during execution and excludes only the faulty components of the affected FUs. Our main contributions are: i) extension of the FUs with BuiltIn Current Sensors (BICS) [10], which monitor the induced transient currents to detect a fault; ii) the run-time instruction scheduling that excludes only the faulty components of the affected FUs exploring mitigation solutions in the current and the next instruction execution; and iii) extensive fault injection simulations varying the number of total and concurrently occurring faults. The obtained results show that the proposed approach mitigates several multiple faults with low overheads.

The organization of this paper is as follows. Section II presents an overview of the approach and the architecture of the proposed coarse-grained mechanism is detailed. Section III presents the experimental results and Section IV concludes this work.

\section{Proposed Run-time Mitigation MECHANism}

We use the 4-issue heterogeneous VLIW data-path of Fig. 1 to schematically illustrate our approach. In this example, the VLIW consists of a 3-stage pipeline with Fetch (F), Decode (DC) and Execute/Memory/Write-Back (EX/MEM/WB). A number of instructions is formatted as one big instruction, named as instruction bundle, which is executed in parallel by the FUs of the processor. The first issue is configured with 1 Arithmetic Logic Unit $(A L U)$ and 1 Branch unit $(B R)$, the second issue with $1 A L U$ and 1 Memory unit (MEM), and the third and fourth issues with $1 A L U$ and 1 Multiplication unit $(M U L)$. The components in gray color correspond to the basic architecture and the components with green color are the extra hardware components required by our approach. The proposed approach focuses on multiple soft faults occurring in the arithmetic FUs, as they have the largest area footprint of the combinatorial components (see section III.A)). The faults in the storage components, e.g. register file, memory 
and pipeline registers, are assumed to be protected with other methods, such as Error Correction Codes (ECC).

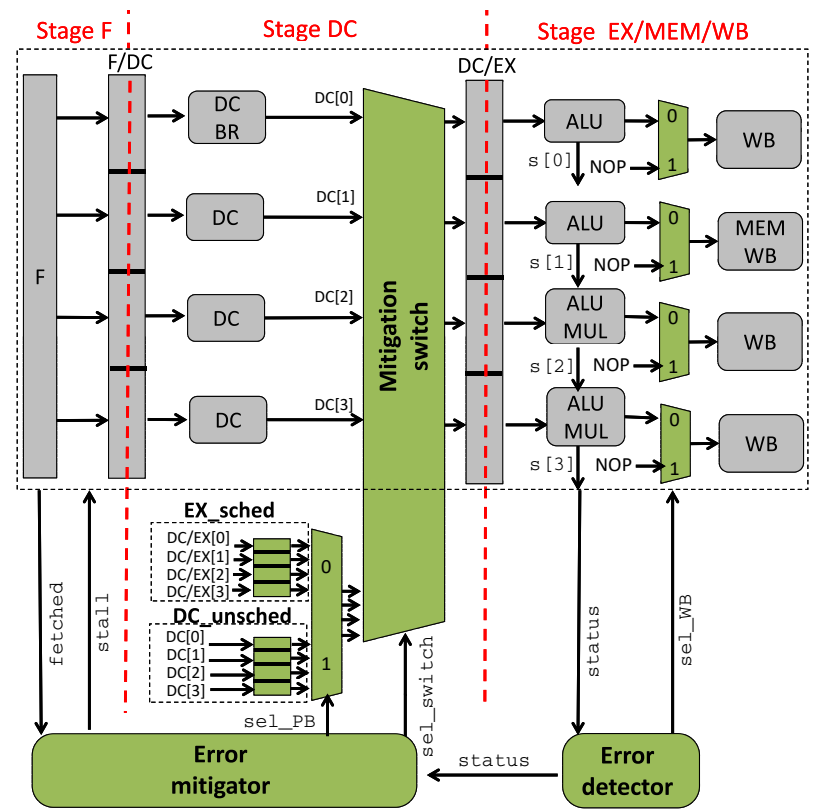

Fig. 1: Extension of the proposed mechanism on the original VLIW (4-issue VLIW example).

\section{A. Overview and motivation}

We will use the following example to present the idea of our mechanism. Fig. 2(a) depicts the original schedule. The instruction bundle is made of 3 additions $\left(A D D_{1}, A D D_{2}, A D D_{3}\right)$ and 1 multiplication $\left(M U L_{1}\right)$. To be able to detect an error, existing software approaches require to duplicate the instructions, such as in Fig. 2(b) and compare the results, and, thus, insert additional time slots increasing the application execution time. Existing hardware approaches are applied for permanent errors, whereas error detection is performed before the application execution, such as [6]. In case an error is detected, for instance if a fault occurs in the MUL unit of the third issue, hardware approaches insert an additional time slot and re-execute the instruction scheduled in the third issue to another compatible FU of another issue, as depicted in Fig. 2(c).

To reduce the negative impact on performance of existing approaches, we propose a mechanism that removes the need of replicated instructions and explores at run-time the rescheduling of the faulty instructions to explore the healthy and idle FUs of the current bundle and the upcoming bundle, as depicted in Fig. 2(d). In this example, the instruction $A D D_{3}$ is moved to the third issue whose $A L U$ is still healthy, whereas the instruction $M U L_{1}$ is moved to fourth issue. Therefore, no need exists for an additional time slot in this example.

\section{B. Error detector}

The error detector keeps the faulty status of the FUs and takes care of miscalculated results. Each FU is internally

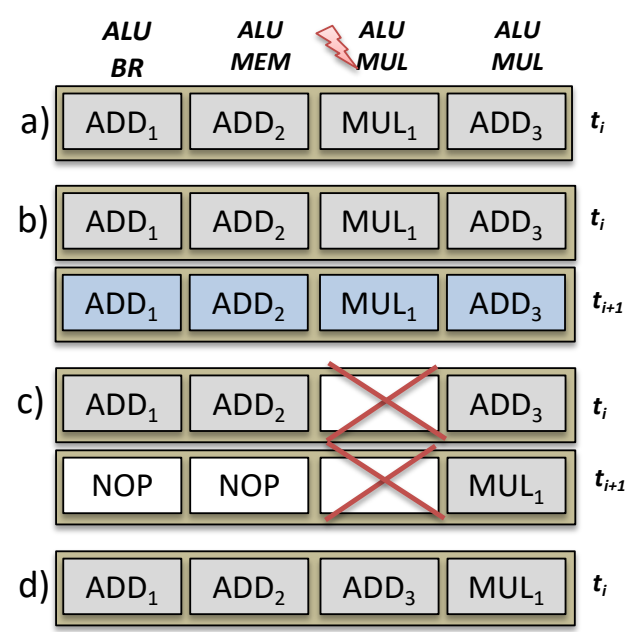

Fig. 2: Example: Motivation and overview of the approach.

enhanced with BICS sensors [10], [11]. A BICS is attached to a group of transistors. During normal operation, the current in the bulk of these transistors is approximately zero. Only the leakage current flows through the biased junction, which is still very low compared to the current generated by energetic particles. When an energetic particle generates a current in the bulk, the bulk-BICS captures that a transient fault occurs. The bulk-BICS has a reset mechanism that allows the fault detection to be active only as long as it takes to dissipate the transitory energy pulse. When the fault has vanished, the affected transistors can be used once again. A signal from the BICS from each FU of one issue is combined into a status signal, $\mathrm{s}[\mathrm{i}]$ in Fig. 1, with a size equalling to the number of FUs of the issue $i$. Hence, if a bit in the signal $\mathrm{s}[i]$ is set, it means the corresponding FUs is affected by a fault. In case of one or more faults at cycle $k-1$, the results of the corresponding instruction(s) of the execution stage are miscalculated, and, thus, they must not be committed. For this purpose, each VLIW issue is enhanced with a multiplexer controlled by the signal sel_WB (with a size equalling to the number of VLIW issues) computed by the error detector. When a bit in sel_WB is set, the corresponding multiplexer passes a NOP result (instead of the miscalculated result) and the WB and MEM enable of the corresponding issue is disabled.

\section{Mitigation switch}

At cycle $k$, the DC_unsched shadow register stores the decoded instructions that couldn't be scheduled and the $E X \_s c h e d$ shadow register keeps the instructions executed but in which an error occurred during their execution. Therefore, at cycle $k$ the instructions to be scheduled can potentially come from three inputs: 1) the decoded instructions at cycle $k-1$ (DC) 2) the remaining instructions not scheduled at cycle $k-1$ (DC_unsched register) and 3) the executed instructions with a fault at cycle $k-1$ (EX_sched register). A mitigation switch is required to move at run-time the instructions in different issues than the ones defined by the compiler's original schedule at 
compile time. We reduce the complexity of the mitigation switch by performing hierarchical decisions. The first decision is whether a shadow register must be used or the currently decoded instructions and the second decision is which shadow register should be used (EX_sched or DC_unsched).

\section{Error Mitigator}

The error mitigator extracts from the fetch stage the required information and the dependencies between bundles and decides which of the three potential inputs that provide instructions for execution ( $D C, D C \_$unsched, and $\left.E X \_s c h e d\right)$ will be scheduled based on the status of the FUs and the type of the instructions.

The mitigator initially performs an early decoding of the instructions at the fetch stage to find the opcode, the destination registers and the source registers of each instruction and to identify instruction dependencies between two consecutive bundles.

To perform the mitigation process, each potential instruction input to the mitigation switch is modeled by a bit mask. A bit mask corresponds to the instruction scheduled at position $i$, as depicted in Fig. 3. a) bit 3: when this bit is set, the FU component required for the instruction execution is an ALU FU; b) bit 2: when this bit is set, the FU component required for the instruction execution is a MUL FU; c) bit 1 : when this bit is set, it means the instruction is an instruction from a previous bundle that has not been scheduled yet; and d) bit 0 : when this bit is set, it means the instruction has a dependency with at least one of the instructions of the next bundle. Then, each of the three potential inputs of the switch is represented by an array of bit masks (EX_sched_mask, DC_unsched_mask and $D C \_$mask) that has a size equalling to the number of VLIW issues.

The status of all FUs components is represented by the array status, where each element is the s signal of an issue enhanced with an additional bit that is set when the issue is occupied as shown in Fig. 3 (bit 0).

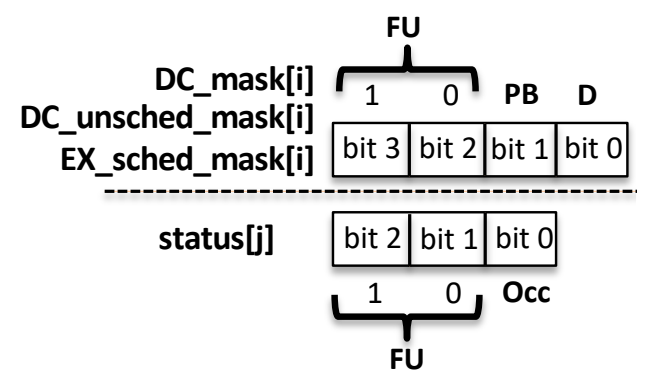

Fig. 3: Bit masks to model the inputs and FU component status of the mitigation switch.

The mitigator decides which of the three potential inputs is to be scheduled next based on the fault occurrences, as depicted by state machine diagram of Fig. 4 , where $i \in[0, n]$ and $n$ is the number of issues.

When at least one new fault occurred at cycle $k-1$ (NF), the executed instructions on the faulty FUs at cycle $k-1$

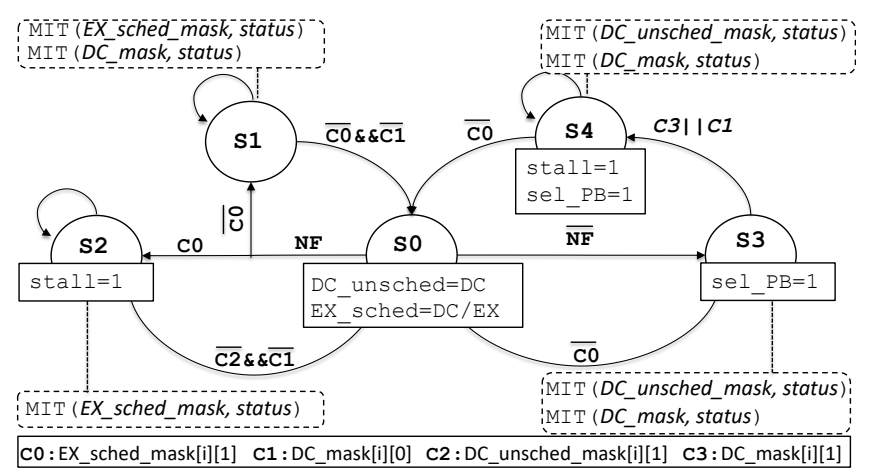

Fig. 4: Functionality of error mitigator.

(which reside in EX_sched register) have to be executed again at cycle $k$.

(S1): If the faulty instructions are decoded instructions at cycle $k-2(\mathrm{CO}==0)$, no stall is required. The array EX_sched_mask is used by the mitigation process, so as to schedule these instructions for execution at cycle $k$. Then, the decoded instructions at cycle $k-1$ are explored by passing the array $D C \_$mask to the mitigation process.

$(S 2)$ : If the faulty instructions are coming from a previous decoded bundle at cycle $k-3,(\mathrm{CO}==1)$, then the stall signal is activated and a new cycle is inserted for the reexecution of these instructions. The array EX_sched_mask is used as an input to the mitigation process. The process is repeated until all instructions are executed.

(S3): If no new fault occurred at cycle $k-1$, the mechanism schedules first the remaining decoded instructions from cycle $k-2$ for execution at cycle $k$. To do so, the array DC_unsched_mask is used as input to the mitigation process. Then, the current decoded instructions DC_mask are used as input to the mitigation process.

(S4): If there are still remaining instructions from the previous bundle and/or if there is any dependent instruction in current decoded instructions that cannot be scheduled, the stall signal is activated to stall the fetch and the decode stage for one cycle, so as to schedule these instructions.

The inputs of the mitigation process are: 1) the mask of the input signal to be scheduled, input; and 2) the array that represents the status of the FU components, status. The outputs are: 1) the control signal sel_switch of the mitigation switch; 2) the control signal sel_PB of the multiplexer between the shadow registers; 3 ) the updated mask of the input signal; and 4) the updated status. The procedure is given by Alg. 1. For all the instructions $i$ of the input (line 3) and for each issue $j$ described by status (line 4), if the instruction $i$ has not been scheduled and the $j$ issue is unoccupied (line 5), check if the required FU is available (line 6). If this is true, the occupied bit of the corresponding status is set (line 7), the remaining bit of the input is cleared, since the instruction is scheduled (line 8), and the signal sel_switch instructs the switch to pass the instruction currently at issue $i$ to issue $j$ (line 9) and the next instruction is explored. 


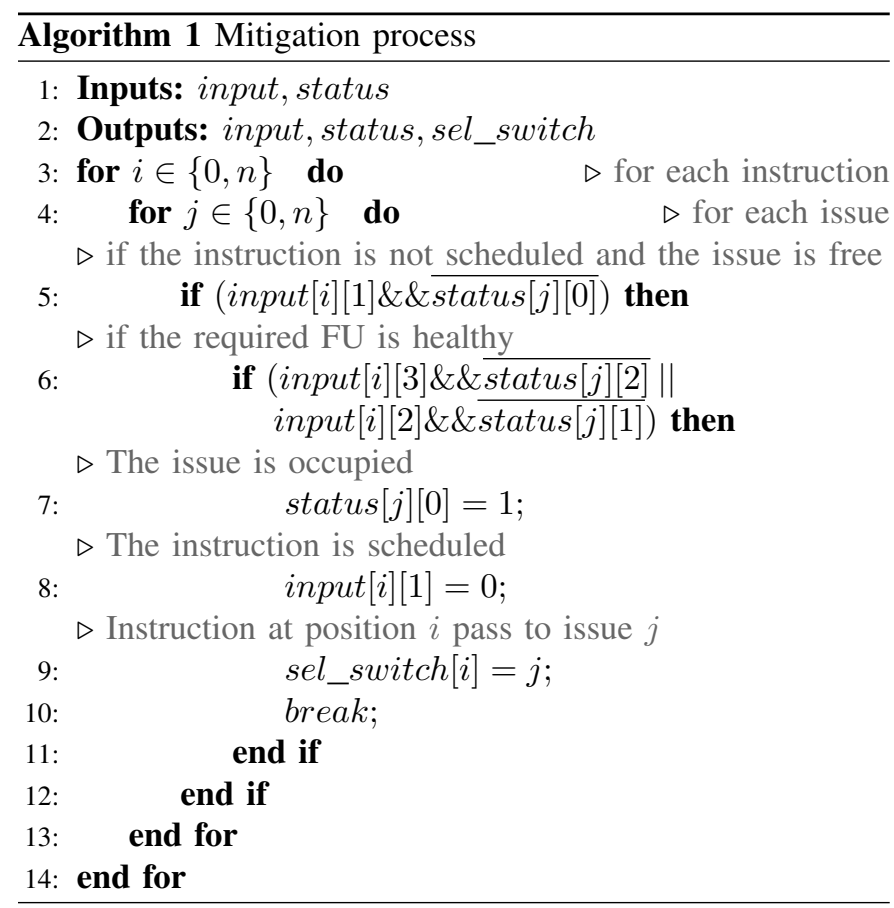

\section{EVALUATION RESULTS}

The original processor and the processor enhanced with the proposed approach have been developed in C++ and synthesized using the Catapult High Level Synthesis (HLS) tool to obtain the RTL design. The gate-level netlist was generated by the Design Compiler tool from Synopsys using $28 \mathrm{~nm}$ ASIC library. To evaluate our approach, we use ten benchmarks from the MediaBench suite [12]. The benchmarks are compiled with VEX compiler [13] for each configuration. For the evaluation results, we used the VEX VLIW processor architecture [14] with two different configurations:

- 4-issue VLIW configured with 4 ALU FUs, 2 MUL FUs, 1 MEM FU and 1 BR FU,

- 8-issue VLIW configured with 8 ALU FUs, 4 MUL FUs, 2 MEM FUs and 1 BR FU.

For every benchmark, Table I shows the average number of useful instructions per bundle, ILP, for both the 4-issue and 8issue VLIW configurations. Because ILP inside bundles is low compared to the number of issues, idle issues of the current or next bundles can be used to execute the faulty instructions in addition to switching instructions with healthy components of units affected by a fault.

\section{A. Area analysis of the unprotected VLIW processor}

First of all, we present the logic area of each unit of the original unprotected VLIW architecture (as depicted in gray color in Fig. 1) in Table II and the area of each pipeline stage for the 4-issue and 8-issue VLIW in Table III. The results motivate the focus of the proposed approach on the execute stage of the VLIW processor, since it covers more area, and, thus, it is more likely to be exposed to faults.
TABLE I: Benchmark characteristics

\begin{tabular}{|r|c|c|}
\hline Benchmark & ILP 4-issues & ILP 8-issues \\
\hline adpcm_dec & 1.77 & 2.28 \\
\hline adpcm_enc & 1.82 & 2.41 \\
\hline bcnt & 2.49 & 3.62 \\
\hline fft32x32s & 2.85 & 4.19 \\
\hline huff & 1.51 & 1.75 \\
\hline motion & 1.94 & 2.39 \\
\hline dct & 2.22 & 3.31 \\
\hline crc & 1.76 & 1.8 \\
\hline fir & 2.09 & 2.5 \\
\hline matrix_mul & 2.61 & 4.46 \\
\hline
\end{tabular}

TABLE II: Area of main VLIW combinatorial units $\left(\mu m^{2}\right)$.

\begin{tabular}{|c|c|c|c|c|}
\hline DC & BR & ALU FU & MUL FU & MEM/WB \\
\hline 250 & 2,290 & 1,533 & 2,310 & 358 \\
\hline
\end{tabular}

\section{B. Performance}

In this paper, we present first experiments to evaluate the benefit of our approach based on coarse-grained FU exploration. The fault model is simplified as much as possible : we randomly injected multiple faults during the benchmarks' execution and, in order to reduce the number of scenarios, we consider the faults as persistent after they occur, i.e. they last for the rest of the execution. Performance overhead is thus the upper bound compared to a transient fault based simulation. Each benchmark is tested for: i) 0 up to 4 multiple faults for the 4-issue configuration; and ii) 0 up to 10 multiple faults for the 8-issue configuration ${ }^{1}$.

Table IV and Table V show the number of execution cycles required to execute the benchmarks considering :

- the original application on the non-protected VLIW (Original),

- the proposed run-time coarse-grained mitigation approach, i.e a time and space idle slot exploitation method in addition to current sensors to use healthy components of faulty function units

- the version where only detection can occur through time and space idle slot exploitation with duplication of the instructions during the execution (2I),

- the version where correction and masking occur through time and space idle slot exploitation with triplication of the instructions during the execution (3I),

- the approach where correction and masking occur by triplicating the FUs in each issue with respect to the original unprotected processor while running the original application (3FUs).

The performance results are obtained by taking the mean value of 20 simulations running the same benchmark and the faults are injected at random execution cycles for each simulation. When no faults occur, the proposed approach have the same performance as the unprotected version, i.e. the number of execution cycles is the same as the original execution. When

\footnotetext{
14 and 10 faults for the 4-issue and 8-issue VLIW configurations respectively, is the maximum number of faults that the approach can sustain at the same time
} 
TABLE III: Area of VLIW stages $\left(\mu m^{2}\right)$.

\begin{tabular}{|c|c|c|}
\hline Stage & 4-issues & 8-issues \\
\hline DC & 3,290 & 6,290 \\
\hline EX & 10,752 & 21,5094 \\
\hline MEM/WB & 1,432 & 2,864 \\
\hline
\end{tabular}

detection and correction occurs by triplicating the FUs in the issues, the number of execution cycles is the same as the original version one (not presented in Table IV and Table V).

From the Table IV, we observe that the average performance overhead of the proposed approach for the 4-issue configuration is $0 \%$ for zero fault up to $83 \%$ for four faults. For the 2I (3I) approach, the average performance overhead is $72 \%$ (152\%) for zero fault up to $262 \%$ (439\%) for four faults. From the Table V, we observe that the average overhead of the proposed approach for the 8-issue configuration is $0 \%$ for zero fault up to $141 \%$ for ten faults. For the 2I (3I) approach the average overhead is $38 \%$ (104\%) for zero fault up to $378 \%$ $(626 \%)$ for ten faults.

Overall, we observe that: 1) the proposed approach inserts significantly lower performance overhead than the approaches with instruction duplication or triplication and 2) in several benchmarks, when the number of faults is low, our approach's performance is very close to the original one, i.e. without faults. The obtained gain of the proposed approach comes from the fact that whenever a persistent fault is detected, the proposed hardware mechanism exploits healthy FUs in the current and the next bundle execution in addition to idle FUs.

\section{Area and delay overhead}

Table VI presents the impact in area and in delay of each component of the proposed run-time coarse-grained mechanism for the 4-issue VLIW. It should be stressed that the delay introduced by the error mitigator does not affect the clock of the VLIW processor, since it is executed in parallel with the VLIW data-path.

TABLE VI: Area and delay overheads introduced by the the components of the proposed mechanism for 4-issue VLIW.

\begin{tabular}{|c|c|c|}
\hline Component & $\begin{array}{c}\text { area } \\
\left(\mu \mathrm{m}^{2}\right)\end{array}$ & $\begin{array}{c}\text { delay } \\
(\mathbf{n s})\end{array}$ \\
\hline Mitigation Switch & 3,918 & 0.16 \\
\hline Error mitigator & 2,112 & 2.44 \\
\hline Error detector & 3,268 & 0.84 \\
\hline
\end{tabular}

Table VII shows the area overhead of the proposed mechanism, the approach that provides error masking by applying triplication of instructions and the approach that triplicates the FUs at each issue. Compared to the unprotected version, the proposed approach implies an area overhead of $18 \%$ and $28 \%$ for the 4-issue and for the 8-issue configurations respectively. For the 3I approach the area overhead is $24 \%$ for the 4 issue configuration while for the 8-issue configurations it is $30 \%$. It is quite similar to the area overhead of the proposed approach. However the proposed approach achieves much better performance as seen in previous section. For the 3FUs approach the area overhead is $45 \%$ for the 4 -issue while for the 8-issue configurations it is $56 \%$.

TABLE VII: VLIW processor area footprint.

\begin{tabular}{|c|c|c|c|c|}
\hline \multirow{2}{*}{ Approach } & \multicolumn{2}{|c|}{ 4-issues } & \multicolumn{2}{c|}{ 8-issues } \\
\cline { 2 - 5 } & $\begin{array}{c}\text { area } \\
\left(\mu \mathrm{m}^{2}\right)\end{array}$ & $\begin{array}{c}\text { area } \\
\mathbf{( \% )}\end{array}$ & $\begin{array}{c}\text { area } \\
\left(\mu \mathrm{m}^{2}\right)\end{array}$ & $\begin{array}{c}\text { area } \\
(\boldsymbol{\%})\end{array}$ \\
\hline Original & 50,844 & - & 79,661 & - \\
\hline Instr. triplication (3I) & 62,812 & 23.54 & 103,598 & 30.05 \\
\hline FUs triplication (3FUs) & 73,523 & 44.60 & 124,137 & 55.83 \\
\hline Proposed mechanism & 60,143 & 18.29 & 102,201 & 28.3 \\
\hline
\end{tabular}

\section{CONCLUSION}

A hardware mechanism is proposed for multiple soft faults that characterizes the FUs of the VLIW processor and reschedules at run-time the instructions, exploiting in time and space idle FUs and healthy components of faulty function units so as to mitigate faulty instructions. From the obtained results, it is shown that several multiple faults can be mitigated with significant reduction in the performance and area overheads compared with approaches that duplicate and triplicate the instructions during execution or triplicate the FUs in the issues. Work in progress is about experiments with faults that last various durations.

\section{REFERENCES}

[1] T. Heijmen, "Radiation-induced soft errors in digital circuits - a literature survey," 2002.

[2] V. Ferlet-Cavrois, P. Paillet and M. Gaillardin et al., "Statistical Analysis of the Charge Collected in SOI and Bulk Devices Under Heavy lon and Proton Irradiation - Implications for Digital SETs," IEEE Transactions on Nuclear Science, pp. 3242-3252, Dec 2006.

[3] J. Klecka, W. Bruckert, and R. Jardine, "Error self-checking and recovery using lock-step processor pair architecture," 2002.

[4] G. Reis, J. Chang, N. Vachharajani, R. Rangan, and D. August, "SWIFT: Software Implemented Fault Tolerance", CGO, pp. 243-254, 2005.

[5] R. Karri et al., "Computer aided design of fault- tolerant application specific programmable processors," $T C$, vol. 49 , no. 11, pp. 1272-1284, Nov 2000.

[6] M. Scholzel and S. Muller, "Combining hardware- and software-based self-repair methods for statically scheduled data paths," DFT, pp. 90-98, Oct 2010.

[7] A. L. Sartor et al., "Exploiting idle hardware to provide low overhead fault tolerance for vliw processors," JETC,vol. 13, no. 2, pp. 13:1-13:21, Jan 2017.

[8] R. Psiakis, A. Kritikakou, and O. Sentieys, "Neda: Nop exploitation with dependency awareness for re- liable vliw processors," ISVLSI, pp. 391-396, July 2017.

[9] R. Psiakis, A. Kritikakou, and O. Sentieys, "Run-time instruction replication for permanent and soft error mitigation in vliw processors," NEWCAS, pp. 321-324, June 2017.

[10] R. Viera, R. Possamai Bastos, J. Dutertre, O. Potin, M.-L. Flottes, G. Di Natale, and B. Rouzeyre, "Validation of Single BBICS Architecture in Detecting Multiple Faults," ATS, Nov 2015.

[11] R. P. Bastos, J. M. Dutertre, and F. S. Torres, "Comparison of bulk built-in current sensors in terms of transient-fault detection sensitivity," VARI, pp. 1-6, Sept 2014.

[12] C. Lee et al., "Mediabench: a tool for evaluating and synthesizing multimedia and communications systems," MICRO, pp. 330-335, Dec 1997.

[13] Hewlett-Packard Laboratories. VEX Toolchain, http://www.hpl.hp.com/downloads/vex/.

[14] J. A. Fisher, P. Faraboschi, and C. Young, "Embedded computing: a VLIW approach to architecture, compilers and tools," Elsevier, 2005. 
TABLE IV: Performance comparison for 4-issue VLIW

\begin{tabular}{|c|c|c|c|c|c|c|c|c|c|c|c|c|c|c|c|c|}
\hline \multirow{2}{*}{ Benchmarks } & \multirow{2}{*}{ Original } & \multicolumn{5}{|c|}{ Proposed mechanism } & \multicolumn{5}{|c|}{ Instr. duplication (2I) } & \multicolumn{5}{|c|}{ Instr. triplication (3I) } \\
\hline & & 0 & 1 & 2 & 3 & 4 & 0 & 1 & 2 & 3 & 4 & 0 & 1 & 2 & 3 & 4 \\
\hline adpcm_dec & 386 & 386 & 412 & 437 & 538 & 565 & 676 & 731 & 809 & 837 & 1124 & 996 & 1150 & 1153 & 1605 & 1682 \\
\hline adpcm_enc & 409 & 409 & 462 & 469 & 503 & 611 & 722 & 831 & 862 & 902 & 1210 & 1062 & 1094 & 1230 & 1387 & 1817 \\
\hline bcnt & 478 & 478 & 511 & 512 & 784 & 1149 & 870 & 904 & 904 & 1199 & 2308 & 1264 & 1297 & 1297 & 1989 & 3364 \\
\hline$f f t 32 \times 32$ & 569 & 569 & 758 & 775 & 932 & 1360 & 1097 & 1290 & 1568 & 1687 & 2710 & 1654 & 1943 & 2052 & 2600 & 4002 \\
\hline huff & 1101 & 1101 & 1136 & 1137 & 1177 & 1491 & 1668 & 1815 & 1895 & 1896 & 2830 & 2348 & 2349 & 2920 & 2920 & 4167 \\
\hline motion & 344 & 344 & 350 & 392 & 619 & 621 & 548 & 549 & 656 & 716 & 1211 & 813 & 868 & 868 & 1110 & 1817 \\
\hline$d c t$ & 1288 & 1288 & 1353 & 1626 & 1844 & 2607 & 2533 & 2662 & 3314 & 3428 & 5173 & 3779 & 3972 & 4962 & 5231 & 7752 \\
\hline crc & 12228 & 12228 & 12274 & 12275 & 14851 & 20972 & 15978 & 15979 & 18896 & 31420 & 41431 & 22600 & 22646 & 22647 & 45847 & 61887 \\
\hline fir & 6852 & 6852 & 8053 & 8594 & 8715 & 11595 & 11780 & 12802 & 14903 & 15564 & 23063 & 16890 & 20491 & 21031 & 24154 & 34520 \\
\hline mat_mul & 11142 & 11142 & 15015 & 16039 & 16359 & 21597 & 20968 & 24841 & 25961 & 31084 & 43178 & 30795 & 34668 & 43884 & 47400 & 64733 \\
\hline \multicolumn{2}{|c|}{ average overhead } & $0.0 \%$ & $12.2 \%$ & $18.4 \%$ & $41.6 \%$ & $82.8 \%$ & $72.5 \%$ & $88.0 \%$ & $111 \%$ & $139 \%$ & $262 \%$ & $152 \%$ & $175 \%$ & $202 \%$ & $277 \%$ & $439 \%$ \\
\hline
\end{tabular}

TABLE V: Performance comparison for 8-issue VLIW.

\begin{tabular}{|c|c|c|c|c|c|c|c|c|c|c|c|c|}
\hline \multirow{2}{*}{ Benchmarks } & \multirow{2}{*}{ Original } & \multicolumn{10}{|c|}{ Proposed mechanism } \\
\cline { 3 - 13 } & & 0 & 1 & 2 & 3 & 4 & 5 & 6 & 7 & 8 & 9 & 10 \\
\hline adpcm_dec & 302 & 302 & 309 & 312 & 312 & 370 & 407 & 433 & 443 & 437 & 471 & 558 \\
\hline adpcm_enc & 323 & 323 & 327 & 339 & 342 & 433 & 405 & 478 & 481 & 489 & 572 & 590 \\
\hline bcnt & 333 & 333 & 334 & 335 & 335 & 336 & 544 & 525 & 674 & 545 & 664 & 1130 \\
\hline fft32x32 & 400 & 400 & 407 & 424 & 479 & 659 & 655 & 738 & 737 & 875 & 1385 & 1388 \\
\hline huff & 951 & 951 & 946 & 946 & 1039 & 959 & 960 & 1039 & 1040 & 1078 & 1113 & 1466 \\
\hline motion & 280 & 280 & 277 & 278 & 284 & 357 & 361 & 358 & 354 & 378 & 415 & 610 \\
\hline$d c t$ & 872 & 872 & 881 & 898 & 968 & 1078 & 1335 & 1369 & 1388 & 1543 & 2573 & 2576 \\
\hline crc & 11955 & 11955 & 11956 & 11956 & 11956 & 11957 & 12217 & 15105 & 12232 & 12516 & 15106 & 20972 \\
\hline fir & 5709 & 5709 & 5890 & 5889 & 5892 & 6072 & 6854 & 6853 & 7750 & 8111 & 8708 & 11814 \\
\hline mat_mul & 6533 & 6533 & 6534 & 6534 & 10619 & 6537 & 10630 & 10950 & 11717 & 14323 & 14833 & 20356 \\
\hline average overhead & $0 \%$ & $1.0 \%$ & $2.2 \%$ & $4.6 \%$ & $17.1 \%$ & $36.7 \%$ & $42.5 \%$ & $46.2 \%$ & $62.9 \%$ & $94.8 \%$ & $141 \%$ \\
\hline
\end{tabular}

\begin{tabular}{|c|c|c|c|c|c|c|c|c|c|c|c|c|}
\hline \multirow{2}{*}{ Benchmarks } & \multirow{2}{*}{ Original Instr. duplication (2I) } \\
\cline { 3 - 13 } & & 0 & 1 & 2 & 3 & 4 & 5 & 6 & 7 & 8 & 9 & 10 \\
\hline adpcm_dec & 302 & 407 & 432 & 443 & 563 & 702 & 710 & 765 & 762 & 773 & 845 & 1116 \\
\hline adpcm_enc & 323 & 439 & 470 & 478 & 492 & 496 & 756 & 769 & 848 & 897 & 866 & 1278 \\
\hline bcnt & 333 & 547 & 548 & 549 & 549 & 936 & 549 & 933 & 938 & 1022 & 1226 & 2064 \\
\hline fft32x32 & 400 & 656 & 705 & 751 & 783 & 839 & 1015 & 1230 & 1391 & 1509 & 1778 & 2789 \\
\hline huff & 951 & 1038 & 1039 & 1040 & 1075 & 1076 & 1610 & 1605 & 1764 & 1744 & 1830 & 2800 \\
\hline motion & 280 & 361 & 383 & 383 & 551 & 384 & 558 & 558 & 561 & 655 & 715 & 1226 \\
\hline dct & 872 & 1339 & 1432 & 1448 & 2450 & 2490 & 1776 & 2547 & 2685 & 2949 & 3429 & 5131 \\
\hline crc & 11955 & 12230 & 12231 & 12231 & 12515 & 12517 & 16219 & 16216 & 16231 & 18908 & 41344 & 41382 \\
\hline fir & 5709 & 6853 & 7754 & 7934 & 7935 & 8115 & 10515 & 13697 & 13812 & 14533 & 15488 & 23505 \\
\hline mat_mul & 6533 & 10950 & 11719 & 11719 & 11719 & 19904 & 19899 & 20520 & 22076 & 22851 & 28517 & 40736 \\
\hline average overhead & $38 \%$ & $46 \%$ & $48 \%$ & $71 \%$ & $96 \%$ & $108 \%$ & $143 \%$ & $155 \%$ & $174 \%$ & $225 \%$ & $378 \%$ \\
\hline
\end{tabular}

\begin{tabular}{|c|c|c|c|c|c|c|c|c|c|c|c|c|}
\hline \multirow{2}{*}{ Benchmarks } & \multirow{2}{*}{ Original } & \multicolumn{10}{|c|}{ Instr. triplication (3I) } \\
\cline { 3 - 13 } & & 0 & 1 & 2 & 3 & 4 & 5 & 6 & 7 & 8 & 9 & 10 \\
\hline adpcm_dec & 302 & 612 & 619 & 616 & 836 & 761 & 1037 & 1127 & 1154 & 1587 & 1295 & 1692 \\
\hline adpcm_enc & 323 & 662 & 675 & 725 & 682 & 1049 & 1064 & 1204 & 1294 & 1280 & 1486 & 1899 \\
\hline bcnt & 333 & 764 & 782 & 782 & 783 & 1389 & 781 & 1417 & 1413 & 1422 & 1954 & 3449 \\
\hline fft32x32 & 400 & 996 & 1029 & 1066 & 1085 & 1399 & 1830 & 1862 & 2077 & 4073 & 2745 & 4311 \\
\hline huff & 951 & 1519 & 1520 & 1521 & 1533 & 1593 & 1635 & 2268 & 2274 & 2832 & 2828 & 4085 \\
\hline motion & 280 & 496 & 497 & 502 & 597 & 603 & 807 & 790 & 828 & 1112 & 1117 & 1750 \\
\hline$d c t$ & 872 & 2136 & 2225 & 2218 & 2506 & 3566 & 2727 & 3900 & 4024 & 4286 & 5149 & 7626 \\
\hline crc & 11955 & 15962 & 15963 & 15978 & 15980 & 15980 & 16979 & 20019 & 22906 & 22914 & 60038 & 61808 \\
\hline fir & 5709 & 10877 & 11179 & 11179 & 11360 & 16219 & 17121 & 19814 & 19939 & 20584 & 24064 & 35147 \\
\hline mat_mul & 6533 & 16408 & 16409 & 16410 & 16411 & 29433 & 30912 & 30870 & 33218 & 33502 & 43379 & 61015 \\
\hline average overhead & $104 \%$ & $108 \%$ & $110 \%$ & $124 \%$ & $200 \%$ & $205 \%$ & $259 \%$ & $277 \%$ & $362 \%$ & $404 \%$ & $626 \%$ \\
\hline
\end{tabular}

